\section{Class 2 integrons in Vibrio cholerae}

Vibrio cholerae is the causative agent of cholera, a potentially epidemic and life-threatening disease. Although $V$. cholerae $\mathrm{O} 1$ and $\mathrm{O} 139$ remain the only known causes of cholera, several recent reports suggest that non-O1, non-O139 strains are increasingly being associated with some epidemic diarrhoeas, including cholera-like diarrhoea (Dalsgaard et al., 1995; Sharma et al., 1998).

While replacement of fluids and electrolytes remains the cornerstone of the management of cholera, antimicrobial therapy can significantly shorten the duration of diarrhoea, and reduce stool volume and requirements for rehydration fluids (Hossain et al., 2002). The rapid emergence of multidrug-resistant strains of $V$. cholerae is considered an issue of great global significance for public health. Integrons play a major role in the spread of antibiotic resistance genes in Gram-negative bacteria, and there are many reports documenting the detection and characterization of class 1 integrons in different serotypes of $V$. cholerae (Dalsgaard et al., 2001; Thungapathra et al., 2002). However, nothing is known about the incidence of class 2 integrons in this important bacterium. Class 2 integrons are well known to be associated with $\operatorname{Tn} 7$, which usually carries three conserved resistance-gene cassettes, $d f r A 1$, sat 1 and $\operatorname{aad} A 1$, that confer resistance to trimethoprim, streptothricin and streptomycin/spectinomycin, respectively (Hansson et al., 2002). This study aimed to investigate the role of class 2 integrons in mediating antibiotic resistance in V. cholerae.

A total of 77 isolates of $V$. cholerae, including members of the O1, O139 and non-O1, non-O139 serogroups isolated in India and Bangladesh from 2001 to 2003, were selected and analysed for the presence of class 2 integrons. For the preliminary detection of class 2 integrons, PCR was performed with the primer pair hep74 and hep51, which is specific to the conserved regions of class 2 integrons, as described elsewhere (DeLappe et al., 2003). Two other primers were then designed according to the preliminary DNA sequencing results for class 2 integrons. These primers were located within the PCR fragment, and were used for complete sequencing of both DNA strands of the whole class 2 integron segment. The nucleotide sequence of the $V$. cholerae class 2 integron has been assigned GenBank accession number AB199789. PCR results showed that only two isolates of $V$. cholerae non-O1, non-O139 were positive (data not shown). One was a clinical isolate (RC121, O27 serotype) isolated in October 2001 in India, and the other was an environmental isolate (B0320, O39 serotype) isolated in July 2003 in Bangladesh. The identity of these two strains as $V$. cholerae was confirmed by species-specific PCR using primers targeted to the gene for the outer-membrane protein OmpW, as described elsewhere (Nandi et al., 2000). All V. cholerae O1 and O139 serotypes were negative for class 2 integrons. The sequencing results of the PCR amplicons revealed the presence of typical class 2 integrons with the three conserved resistance gene cassettes $d f r A 1$, sat 1 and aadA1. To investigate the role of class 2 integrons in mediating the antibiotic-resistance phenotype in these two positive strains, MICs of various antibiotics were determined by the broth microdilution method, as described elsewhere (Dalsgaard et al., 2001). MIC results showed that both strains had a multidrug-resistance (MDR) phenotype to types of antibiotics, including trimethoprim, streptomycin and spectinomycin, whose resistances may be encoded by class 2 integron gene cassettes (Table 1). These results confirm the role of class 2 integrons in mediating part of the MDR phenotype of these two strains.

With respect to the location of the class 2 integrons, even though several plasmids of different sizes were detected in RC121, O27 serotype, none was detected in B0320, O39 serotype (data not shown). Hence, Southern blot hybridization using the whole class 2 integron as a probe was carried out to determine the location of the class 2 integron in RC121, O27 serotype. The results showed that no signal was detected corresponding to any of the plasmids (data not shown). Therefore, the class 2 integrons in both serotypes may be located in the chromosome.

In the past, $V$. cholerae strains of serotypes other than $\mathrm{O} 1$ and $\mathrm{O} 139$ have attracted little
Table 1. MICs of antibiotics for $V$. cholerae strains RC121, O27 serotype, and B0320, О39 serotype

\begin{tabular}{|lcc|}
\hline \multirow{2}{*}{ Antibiotic } & \multicolumn{2}{c|}{ MIC $\left(\mathbf{m g ~ l}^{-\mathbf{1}}\right)$} \\
\cline { 2 - 3 } & $\mathbf{R C 1 2 1 , \mathbf { O 2 7 }}$ & $\mathbf{B 0 3 2 0 , \mathbf { O 3 9 }}$ \\
\hline Streptomycin & 128 & 128 \\
Spectinomycin & 128 & 64 \\
Trimethoprim & 32 & 64 \\
Ampicillin & 128 & 32 \\
Nalidixic Acid & 64 & 64 \\
Ciprofloxacin & 32 & 2 \\
Gentamicin & 2 & $0 \cdot 5$ \\
Chloramphenicol & 16 & 4 \\
Tetracycline & 4 & $0 \cdot 5$ \\
Kanamycin & 8 & 2 \\
Neomycin & 4 & 4 \\
Cefotaxime & $0 \cdot 25$ & $0 \cdot 125$ \\
\hline
\end{tabular}


scientific attention, but recently, $V$. cholerae non-O1, non-O139 strains have been implicated in many cholera-like epidemics (Dalsgaard et al., 1995; Sharma et al., 1998). Also, the emergence of a new $V$. cholerae O139 serogroup, as a second aetiologic agent of cholera epidemics, is believed to have arisen by horizontal gene transfer from serogroup O1 to a non-O1 serogroup (Faruque et al., 2003). Furthermore, the sharp distinction between epidemic and non-epidemic strains of $V$. cholerae has now become more complicated, following the discovery of horizontal and vertical genetic transfer of mobile genetic elements among different $V$. cholerae serotypes (Faruque et al., 2003). Therefore, researchers working on cholera are directing attention not only to the epidemic strains of $V$. cholerae $\mathrm{O} 1$ and $\mathrm{O} 139$, but also to non-O1, non-O139 strains. Hence, detection of class 2 integrons in $V$. cholerae non-O1, non-O139 is considered a step towards a better understanding of the mechanisms and evolution of the recently acquired MDR phenotype in many epidemic strains of V. cholerae.

\section{Acknowledgements}

We are grateful to Dr S. Shinoda for kindly providing the $V$. cholerae strains used in this study. We also thank Dr E. Arakawa for the determination of the serotypes. This work was supported by a Grant-in-Aid for scientific research to T.S. from the Ministry of Education, Culture, Sports, Science and Technology of Japan.

\section{Ashraf M. Ahmed, $\uparrow$ Fumio Kawaguchi and Tadashi Shimamoto}

Laboratory of Food Microbiology and Hygiene, Graduate School of Biosphere Science, Hiroshima University, HigashiHiroshima, Hiroshima 739-8528, Japan

†Present address: Department of Microbiology, Faculty of Veterinary Medicine, Tanta University, Kafr El-Sheikh 33516 , Egypt.

Correspondence: Tadashi Shimamoto (tadashis@hiroshima-u.ac.jp)

Dalsgaard, A., Albert, M. J., Taylor, D. N., Shimada, T., Meza, R., Serichantalergs, O. \& Echevarria, P. (1995). Characterization of Vibrio cholerae non-O1 serogroups obtained from an outbreak of diarrhea in Lima, Peru. J Clin Microbiol 33, 2715-2722.

Dalsgaard, A., Forslund, A., Sandvang, D., Arntzen, L. \& Keddy, K. (2001). Vibrio cholerae O1 outbreak isolates in Mozambique and South Africa in 1998 are multiple-drug resistant, contain the SXT element and the aadA2 gene located on class 1 integrons. J Antimicrob Chemother 48, 827-838.

DeLappe, N., O'Halloran, F., Fanning, S., Corbett-Feeney, G., Cheasty, T. \& Cormican, M. (2003). Antimicrobial resistance and genetic diversity of Shigella sonnei isolates from western Ireland, an area of low incidence of infection. J Clin Microbiol 41, 1919-1924.

Faruque, S. M., Sack, D. A., Sack, R. B., Colwell, R. R., Takeda, Y. \& Nair, G. B.

(2003). Emergence and evolution of Vibrio cholerae O139. Proc Natl Acad Sci U S A 100, 1304-1309.

Hansson, K., Sundstrom, L., Pelletier, A. \& Roy, P. H. (2002). IntI2 integron integrase in $\operatorname{Tn} 7$. J Bacteriol 184, 1712-1721.

Hossain, M. S., Salam, M. A., Rabbani, G. H., Kabir, I., Biswas, R. \& Mahalanabis, D. (2002). Tetracycline in the treatment of severe cholera due to Vibrio cholerae O139 Bengal. J Health Popul Nutr 20, 18-25.

Nandi, B., Nandy, R. K., Mukhopadhyay, S., Nair, G. B., Shimada, T. \& Ghose, A. C. (2000). Rapid method for species-specific identification of Vibrio cholerae using primers targeted to the gene of outer membrane protein OmpW. J Clin Microbiol 38, 4145-4151.

Sharma, C., Thungapathra, M., Ghosh, A. \& 11 other authors (1998). Molecular analysis of non-O1, non-O139 Vibrio cholerae associated with an unusual upsurge in the incidence of cholera-like disease in Calcutta, India. J Clin Microbiol 36, 756-763.

Thungapathra, M., Amita, Sinha, K. K., Chaudhuri, S. R., Garg, P., Ramamurthy, T., Nair, G. B. \& Ghosh, A. (2002). Occurrence of antibiotic resistance gene cassettes $a a c\left(6^{\prime}\right)-I b$, $d f r A 5, d f r A 12$, and ereA2 in class I integrons in non-O1, non-O139 Vibrio cholerae strains in India. Antimicrob Agents Chemother 46, 2948-2955. 